\title{
WOMEN IN TRANSPLANTATION (WIT): CAPÍTULO BRASILEIRO
}

\author{
Women in Transplantation (WIT): Brazilian Chapter \\ Érika Bevilaqua Rangel'1, Lígia Camera Pierrotti², Bartira Aguiar Roza ${ }^{3}$, Janine Schirmer ${ }^{3}$, \\ Lilian Monteiro Pereira Palma ${ }^{4}$, Marilda Mazzali ${ }^{5}$, Raquel Silveira Bello Stucchi ${ }^{6}$, Wanessa Trindade Clemente ${ }^{7}$, \\ Ilka de Fatima Santana Ferreira Boin ${ }^{8}$, Maria Cristina Ribeiro de Castro $^{9}$
}

\section{RESUMO}

Introdução: A participação das mulheres nos diversos segmentos profissionais tem aumentado nas últimas décadas. No entanto, a transição para cargos de liderança apresenta algumas barreiras, de modo que planos estratégicos têm sido propostos em vários estudos. Métodos: Descrever a experiência da organização Women in Transplantation (WIT) / The Transplantation Society (TTS), fundada em 2009, nos Estados Unidos, além de analisar outros estudos que investigaram a equidade de gêneros nas diversas áreas profissionais. Resultados: A WIT vem desenvolvendo atividades durante todos os anos, após sua fundação, e estabeleceu dois pilares: (i) avançar e inspirar profissionais mulheres nas áreas do transplante; (ii) defender questões relacionadas ao sexo e gênero na área do transplante. Similarmente, os capítulos WIT australiano e neozelandês e The Initiative on Women in Science and Engineering Working Group propuseram identificar possíveis problemas de equidade de gênero, conceber e implementar estratégias e intervenções potenciais para enfrentar esses desafios e estabelecer metas realistas e alcançáveis para melhorar e facilitar a igualdade, a equidade e a diversidade de gênero no transplante. Mais de $60 \%$ das mulheres acreditam que flexibilidade no trabalho, mentoria e treinamento para liderança são aspectos chaves para o desenvolvimento da carreira profissional, além do estabelecimento de uma network robusta. Conclusão: Nosso grupo de trabalho inspirou-se nessas iniciativas e discutiu estratégias para verificar o perfil profissional das mulheres que trabalham nas diversas áreas do transplante e da doação de órgãos no Brasil. Observamos uma oportunidade para identificar possíveis barreiras durante o desenvolvimento de suas carreiras e transição para liderança. Intencionamos, portanto, fundar o Capítulo Brasileiro WIT no próximo Congresso da Associação Brasileira de Transplante de Órgãos (ABTO), em outubro de 2019, na cidade de Campinas, São Paulo, Brasil, além de propor que seja criado um comitê acessório da WIT junto à ABTO.

Descritores: Equidade de Gênero; Mulheres Trabalhadoras; Transplante.

\section{Instituições:}

${ }^{1}$ Disciplina Nefrologia, Transplante de Pâncreas-Rim, Universidade Federal de São Paulo; Instituto de Ensino e Pesquisa, Hospital Israelita Albert Einstein, São Paulo, SP, Brasil

2 Divisão de Doenças Infecciosas, Hospital das Clínicas, Faculdade de Medicina da Universidade de São Paulo, São Paulo, SP, Brasil

${ }^{3}$ Escola Paulista de Enfermagem, Universidade Federal de São Paulo, São Paulo, SP, Brasil

${ }^{4}$ Disciplina Nefrologia Pediátrica, Transplante Renal Pediátrico, Universidade Estadual de Campinas, Campinas, SP, Brasil

${ }^{5}$ Disciplina Nefrologia, Transplante Renal, Faculdade de Ciências Médicas, Universidade Estadual de Campinas, Campinas, SP, Brasil

${ }^{6}$ Disciplina Infectologia, Faculdade de Ciências Médicas, Universidade Estadual de Campinas, Campinas, SP, Brasil

${ }^{7}$ Departamento de Propedêutica Complementar da Faculdade de Medicina, Universidade Federal de Minas Geais (UFMG); Transplante Hepático do Hospital das Clínicas da UFMG, Belo Horizonte, MG, Brasil
${ }^{8}$ Departamento de Cirurgia, Transplante Hepático, Faculdade de Ciências Médicas, Universidade Estadual de Campinas, Campinas, SP, Brasil

${ }^{9}$ Disciplina de Nefrologia, Transplante Renal, Faculdade de Medicina da Universidade de São Paulo, São Paulo, SP, Brasil

\section{Correspondência:}

Érika Bevilaqua Rangel

Disciplina de Nefrologia, Universidade Federal de São Paulo,

Rua Botucatu 590, $15^{\circ}$ andar, CEP 04023-062, São Paulo/SP, Brasil Tel.: (11) 5904-1699

E-mail: erikabr@uol.com.br 


\section{INTRODUÇÃO}

A organização WIT (Women in Transplantation) e o primeiro Comitê de Direção foram estabelecidos em 2009, durante a presidência da Professora Dra. Kathryn Wood, na The Transplantation Society (TTS). ${ }^{1}$

$\mathrm{Na}$ ocasião, foram definidas a missão, a visão e as diretrizes da WIT:

\section{Missão:}

Promover e inspirar profissionais mulheres nas áreas do transplante e defender questões de sexo e gênero no transplante.

\section{Visão:}

Buscar a equidade de gênero e a inclusão das mulheres nas diversas áreas do transplante.

\section{Diretrizes:}

(i) Alcançar amplamente a diversidade global, o acesso e a participação das mulheres nas diversas áreas do transplante, os quais são essenciais para alcançar a missão da WIT.

(ii) Ser uma iniciativa independente e aberta à colaboração e parceria entre sociedades e associações profissionais que compartilham a missão e os objetivos da WIT.

(iii) Atuar na defesa, educação, engajamento e conexão entre gêneros, culturas e domínios profissionais nas diversas áreas do transplante, os quais são essenciais para o sucesso e o impacto da WIT.

A partir de 2009, várias estratégias foram conduzidas pela WIT nos diversos eventos relacionados ao transplante de órgãos:

- 2010: Lançamento da pesquisa mundial, com duração de dois anos, visando profissionais mulheres nas áreas DO TRANSPLANTE.

- 2011: Programa Internacional de Mentoria lançado com mais de 40 relacionamentos mentores/mentorados.

- 2012: Pesquisa mundial concluída com mais de 500 profissionais mulheres, em 45 países.

- 2013: Mais de 80 mentoras mulheres assinaram a segunda rodada do Programa Internacional de Mentoria.

- 2014: Participação em eventos da WIT ultrapassa 1.000 participantes mulheres/Prêmio WIT inaugurado no World Transplant Congress, 26 a 31 de julho, São
Francisco, Califórnia, Estados Unidos.

- 2015: Criação de uma biblioteca de recursos on-line para todas as sessões e palestras da WIT.

- 2016: Lançamento do Workshop para Mulheres Líderes em Desenvolvimento.

- 2017: Redefinição da missão e da visão da WIT e introdução dos pilares.

Os pilares estratégicos foram, então, definidos:

\section{Pilar 1}

Avançar e inspirar profissionais mulheres nas diversas áreas do transplante.

(i) Garantir a equidade na liderança profissional das mulheres nas diversas áreas do transplante.

(ii) Aumentar o número de mulheres palestrantes nas reuniões nacionais e internacionais.

(iii) Aumentar os subsídios/prêmios monetários para profissionais mulheres nas áreas do transplante.

(iv) Aumentar o número de mulheres nas áreas do transplante, conectadas e apoiando umas às outras (mentorias formal e informal).

\section{Pilar 2}

Defender questões relacionadas ao sexo e gênero nas diversas áreas do transplante.

(i) Assegurar financiamento sustentável destinado a projetos (coleta de dados, pesquisa, educação) para tratar de questões de sexo e gênero.

(ii) Programar sessões destinadas a questões de sexo e gênero nas principais reuniões nacionais e internacionais de transplante.

(iii) Dobrar a porcentagem de receptoras de transplante de órgãos do sexo feminino (especialmente em países com disparidades).

(iv) Diminuir as disparidades no número de doadores vivos de órgãos dos sexos masculino e feminino em todo o mundo.

Similarmente, a organização WIT, na Nova Zelândia e na Austrália, definiu seus principais objetivos: ${ }^{2}$

(i) Identificar possíveis problemas de equidade de gênero na força de trabalho nas diversas áreas do transplante.

(ii) Conceber e implementar estratégias e intervenções potenciais para enfrentar esses desafios. 
Érika Bevilaqua Rangel, Lígia Camera Pierrotti, Bartira Aguiar Roza, Janine Schirmer, Lilian Monteiro Pereira Palma, Marilda Mazzali, Raquel Silveira Bello Stucchi, Wanessa Trindade Clemente, Ilka de Fatima Santana Ferreira Boin, Maria Cristina Ribeiro de Castro

(iii) Estabelecer metas realistas e alcançáveis para melhorar e facilitar a igualdade, a equidade e a diversidade de gênero no transplante.

Outras iniciativas para promoção e avanço profissional das mulheres foram observadas em outras áreas. Em 2015, a respeitada revista internacional Cell Stem Cell publicou as sete estratégias para o avanço da carreira das mulheres nas áreas de engenharia, ciência e medicina. ${ }^{3}$

Essas estratégias foram propostas pela força-tarefa do The Initiative on Women in Science and Engineering Working Group e estão descritas a seguir:

1) Implementar gastos flexíveis em cuidados familiares. Segundo essa política de gênero neutro, as organizações que concedem subsídios permitiriam que os donatários usassem uma certa porcentagem dos fundos do subsídio para pagar por cuidados com filhos, idosos ou despesas relacionadas à família, a fim de incentivar palestras ministradas pelas convidadas ou mesmo a participação em reuniões e conferências científicas.

2) Promover o Prêmio "Mãos Extras": As "mãos extras" permitiriam que os projetos de pesquisa das pesquisadoras em diversas áreas continuassem progredindo sem interrupções, permitindo o aumento da produtividade nos primeiros anos, que costumam ser os mais críticos, e possibilitando a independência como pesquisadoras.

3) Recrutar comitês externos de revisão e comitês de seleção de palestrantes equilibrados por gênero. O grupo de trabalho IWISE (New York Stem Cell Foundation's Initiative on Women in Science and Engineering) incentiva as organizações a compartilhar amplamente nomes e listas de possíveis revisores e palestrantes para facilitar a identificação pelas organizações. Por exemplo, o Comitê de Mulheres em Biologia Celular da Sociedade Americana de Biologia Celular mantém essa lista (http://ascb.org/wicb-committee/). O Grupo de Trabalho IWISE sugere que, no mínimo, as mulheres devam constituir $20 \%$ de todos os comitês de revisão.

4) Incorporar declarações de tendência implícita. Essas declarações descrevem o conceito de preconceito implícito para os revisores e reiteram o compromisso das organizações com a igualdade de gênero, a igualdade de oportunidades para homens e mulheres e a diversidade, em todas as suas formas, ao longo de seus programas. Como instituição, a New York Stem Cell Foundation, por exemplo, procura promover a igualdade de gênero e aumentar a diversidade, em todas as suas formas, ao longo de seus programas. Estudos demonstraram que, frequentemente, existem tendências sutis, inconscientes e implícitas na ciência acadêmica, que têm o potencial de impactar negativamente os resultados dos processos de revisão. Para esse fim, todos devem estar cientes dos possíveis vieses implícitos ao revisar, pontuar e discutir candidatos e inscrições durante todo o processo de revisão, para que possamos trabalhar juntos para combater seu potencial impacto negativo.

5) Foco na educação como ferramenta. Instituições, doadores e cientistas devem comprometer-se com a educação como uma ferramenta para combater os problemas enfrentados pelas mulheres na Ciência. As instituições acadêmicas devem fazer do treinamento de conscientização de gênero um componente padrão de suas orientações de pós-doutorado e treinar os principais pesquisadores para tomar medidas proativas em seus próprios laboratórios para mitigar as disparidades de gênero.

6) Criar um boletim institucional para verificar, sinalizar e estabelecer medidas para a igualdade de gênero (Tabela 1).

Tabela 1 - Proposta do Relatório Institucional inicial em busca da igualdade de gênero. Adaptado de Smith et al ${ }^{3}$

\begin{tabular}{|l|}
\hline $\begin{array}{l}\text { Qual a proporção de graduandos do seu departamento é do sexo } \\
\text { feminino? }\end{array}$ \\
\hline $\begin{array}{l}\text { Qual a proporção de estudantes de pós-graduação do seu } \\
\text { departamento é do sexo feminino? }\end{array}$ \\
\hline $\begin{array}{l}\text { Que proporção do corpo docente do seu departamento (professor } \\
\text { adjunto, associado ou titular) é do sexo feminino? }\end{array}$ \\
\hline $\begin{array}{l}\text { Nos últimos cinco anos, qual a proporção de professores titulares } \\
\text { de seu departamento que foi recrutado de fora da instituição era do } \\
\text { sexo feminino? }\end{array}$ \\
\hline $\begin{array}{l}\text { Nos últimos cinco anos, qual a proporção de membros do corpo } \\
\text { docente do seu departamento pela primeira vez recrutada de fora da } \\
\text { instituição era do sexo feminino? }\end{array}$ \\
\hline $\begin{array}{l}\text { Qual é a sua política institucional em relação a férias familiares } \\
\text { remuneradas e interrupção temporária para os prazos de entrega } \\
\text { devido a licença maternidade? Existe suporte financeiro adicional } \\
\text { disponível durante a licença maternidade? }\end{array}$ \\
\hline $\begin{array}{l}\text { Qual é a sua política institucional em relação à representação } \\
\text { feminina em comitês internos? Qual é o percentual atual de } \\
\text { representação feminina em comitês de nomeações, promoções, } \\
\text { finanças, prêmios e planos estratégicos? }\end{array}$ \\
\hline $\begin{array}{l}\text { Nos últimos } 12 \text { meses, qual a proporção dos palestrantes do } \\
\text { programa de seminários externos do seu departamento era do sexo } \\
\text { feminino? }\end{array}$ \\
\hline
\end{tabular}

7) Estabelecer parcerias para expandir os bancos de dados de pesquisa existentes e elaborados por mulheres, nos campos da Ciência, Medicina e Engenharia. O Grupo de trabalho IWISE sugere que financiadores, instituições acadêmicas e revistas científicas colaborem com a Organização Europeia de Biologia 
Molecular (http://www.embo.org/science-policy/womenin-science/wils-database-of-women- ciências da vida), a Sociedade Americana de Navegação Científica em Biologia Celular (http://ascb.org/science-navigator/) e outras organizações relevantes para desenvolver ou expandir bancos de dados de pesquisa existentes, incluindo mulheres nas áreas da Ciência, Medicina e Engenharia.

Em2017, a editora Elsevierpublicou uma análise realizada ao longo de 20 anos, em 12 países e envolvendo 27 áreas, conhecida como Gender in the Global Research Landscape. ${ }^{4}$ Como administrador da pesquisa mundial, a Elsevier declarou ter responsabilidade na promoção da igualdade de gênero junto à organização STEM (Science, Technology, Engineering, and Mathematics ou Ciência, Tecnologia, Engenharia e Matemática) e no avanço da compreensão do impacto de gênero, sexo e diversidade na pesquisa. Nesse sentido, a Elsevier declarou também apoiar totalmente o Objetivo 5 do Desenvolvimento Sustentável das Nações de Desenvolvimento, que se refere a "alcançar a igualdade de gênero e capacitar todas mulheres e meninas" e à declaração do Conselho Global de Pesquisa Princípios e Ações que Promovem a Igualdade e Status das Mulheres em Pesquisa.

Os principais achados foram:

(i) A proporção de mulheres entre pesquisadores e inventores está aumentando em todos os 12 países, ao longo do tempo.

(ii) As mulheres publicam menos trabalhos de pesquisa, em média, que os homens, mas não há evidências de que isso afete como seus artigos são citados ou lidos.

(iii) As mulheres são menos propensas que os homens a colaborar internacionalmente em trabalhos de pesquisa.

(iv) As mulheres são um pouco menos propensas que os homens a colaborar entre setores acadêmicos e corporativos de pesquisa.

(v) Em geral, a produção acadêmica das mulheres inclui uma proporção ligeiramente maior de profissionais interdisciplinares de pesquisa do que a dos homens.

(vi) Entre os pesquisadores, as mulheres, geralmente, têm menor mobilidade internacional do que os homens.

(vii) A pesquisa envolvendo gênero está crescendo, em termos de tamanho e complexidade, com novos tópicos surgindo ao longo do tempo. (viii) O antigo domínio dos Estados Unidos na pesquisa de gênero declinou, enquanto a atividade de pesquisa na União Europeia aumentou.

(ix) A proporção de patentes com, pelo menos, uma mulher nomeada como inventora tende a ser maior entre os inventores do que a proporção de mulheres que submetem individualmente uma patente.

Neste relatório, há menção ao Brasil, no qual uma proporção similar de mulheres e homens apresenta publicações na área de pesquisa científica, nas áreas de Medicina e Biológicas, embora haja disparidade na área de Exatas. ${ }^{4}$

A falta de equidade de gêneros também é identificada na área corporativa, segundo uma pesquisa financiada pela empresa JUMP \& Eve-Olution Survey, publicada em março de 2012, envolvendo 400 empresárias na Europa (Figuras 1A e 1B). ${ }^{5}$ Nessa pesquisa, o grupo de mulheres foi entrevistado em 2005 e em 2012.

Os resultados mostram que, desde a primeira etapa da pesquisa realizada em 2005, muito mais mulheres acreditam que seu ambiente corporativo não reconhece que seus pontos fortes e habilidades são diferentes dos colegas do sexo masculino, mas igualmente valiosos para o sucesso da empresa.

Dentre as medidas para incentivar o desenvolvimento da carreira profissional das mulheres, mais de $65 \%$ acham importante ter flexibilidade na jornada de trabalho, mentoria e treinamento profissional (coaching).

Uma pesquisa mais recente, de 2018 ( $n=1075$ mulheres), abordou o tema "liderança" e identificou que a transição para cargos de liderança está associada a situações de alto risco, ainda desafiadoras, para que haja a retenção e o sucesso do processo liderança. ${ }^{6} \mathrm{O}$ estudo identificou barreiras que contribuem para a interrupção da progressão da carreira das mulheres e as ações que podem ser tomadas para removê-las.

Os principais achados foram:

(i) As mulheres sentem-se prontas para assumir posições de liderança.

(ii) As mulheres almejam as mudanças, mas têm medo de cometer erros e sentem-se mais severamente julgadas do que os homens.

(iii) As mulheres confiam em se manifestar, mas acreditam que existe um viés que favorece os homens em termos de remuneração e promoção.

(iv) As mulheres não têm influência para se tornarem líderes porque não têm uma network robusta. 
Érika Bevilaqua Rangel, Lígia Camera Pierrotti, Bartira Aguiar Roza, Janine Schirmer, Lilian Monteiro Pereira Palma, Marilda Mazzali, Raquel Silveira Bello Stucchi, Wanessa Trindade Clemente, Ilka de Fatima Santana Ferreira Boin, Maria Cristina Ribeiro de Castro

Figura 1 - Principais achados da pesquisa Women leaders speak out! (A) e estratégias para permitir o desenvolvimento da carreira profissional das mulheres (B). ${ }^{5}$

A

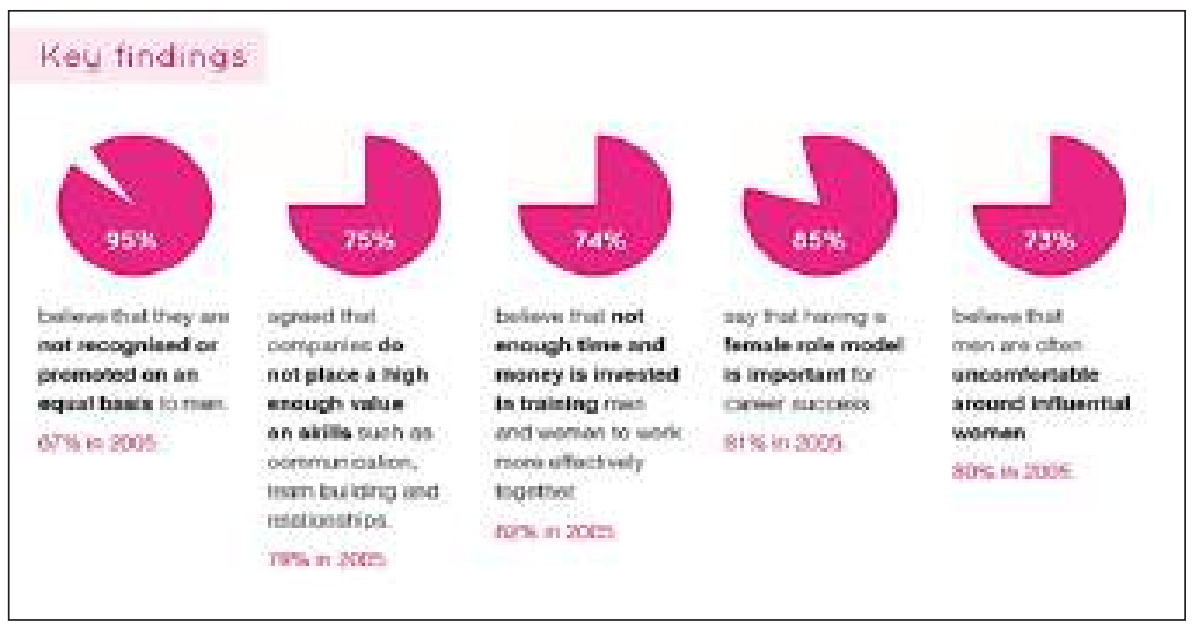

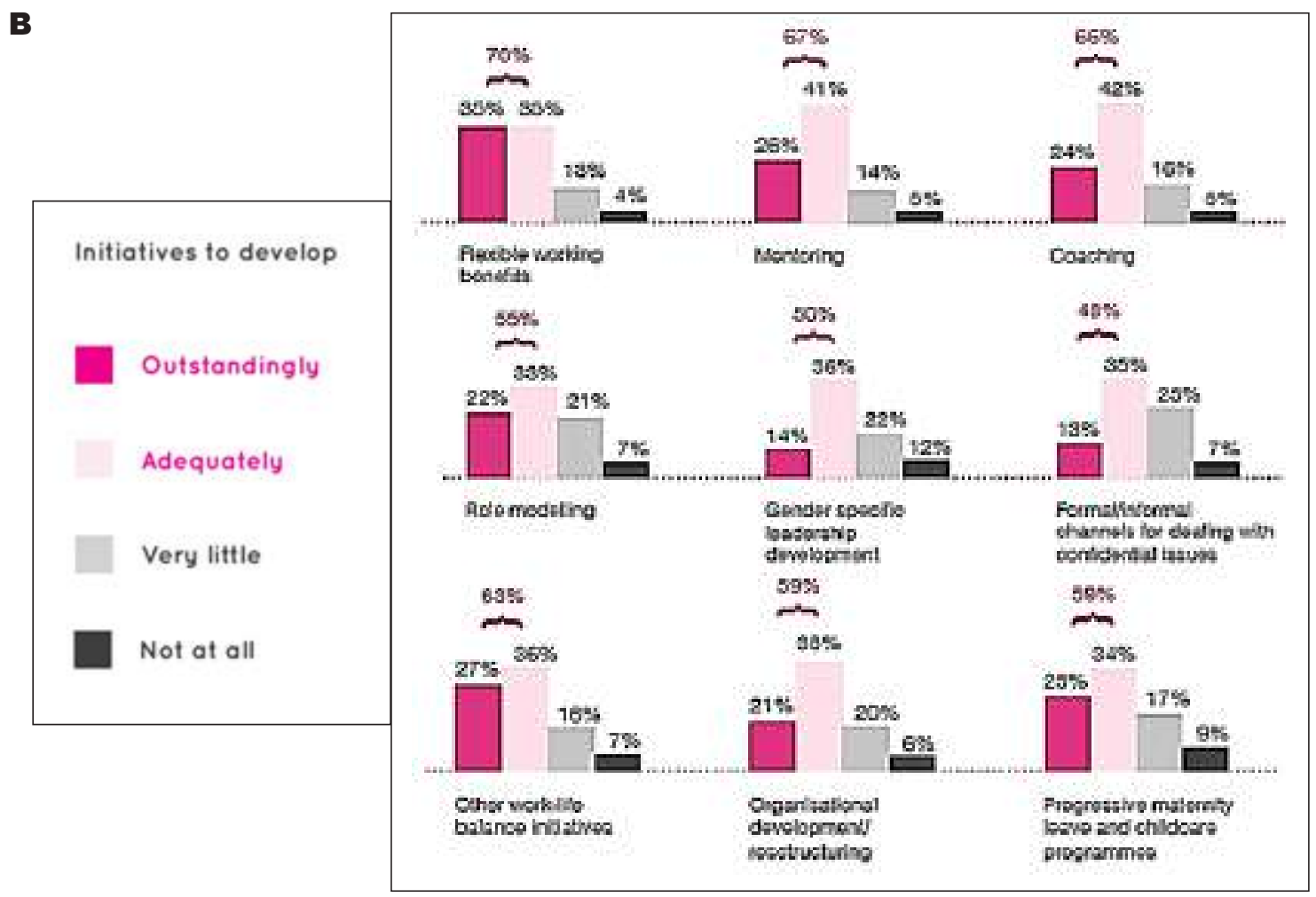




\section{CONCLUSÃO}

Nosso grupo de trabalho foi recentemente criado, a partir da inspiração nas iniciativas previamente descritas e vem discutindo estratégias para verificar o perfil profissional das mulheres que trabalham nas diversas áreas do transplante e da doação de órgãos, no Brasil. Observamos, portanto, uma oportunidade para identificar possíveis barreiras durante o desenvolvimento de suas carreiras e na transição para cargos de liderança, além de realizar um levantamento sobre equidade de gêneros nas diversas áreas do transplante no Brasil.
O desenvolvimento de estratégias direcionadas ao aumento da network, mentoria, treinamento de liderança, dentre outros, são pontos chaves para essas discussões, tendo em vista a importante participação do Brasil no cenário mundial de transplante.

Intencionamos, portanto, fundar o Capítulo Brasileiro Women in Transplantation, no próximo congresso da Associação Brasileira de Transplante de Órgãos (ABTO), em outubro de 2019, na cidade de Campinas, São Paulo, Brasil, além de propor que seja criado um comitê acessório da WIT junto à ABTO.

\section{ABSTRACT}

Introduction: Participation of women in different professional segments has been increasing in recent decades. However, transition to leadership positions is associated to some barriers, so that strategic plans were proposed in several studies. Methods: To describe the experience of the organization Women in Transplantation (WIT)/The Transplantation Society (TTS) founded in 2009 in the United States, as well as registering the results of other studies that investigated gender equity in several fields. Results: WIT has been developing activities over the years since its foundation, and has established 2 pillars: (i) to advance and inspire female transplant professionals; (ii) to advocate issues of sex and gender in transplantation. Similarly, the Australian and New Zealander WIT chapters and The Initiative on Women in Science and Engineering Working Group Similarly, WIT founded in Australia and New Zealand, and The Initiative on Women in Science and Engineering Working Group proposed to identify possible gender equity issues, to design and implement potential strategies and interventions to address some of those challenges and to establish realistic and attainable goals to improve and facilitate gender equality, equity and diversity in transplantation. Above $60 \%$ of women believe that flexibility at work, mentoring and coaching are key aspects for the development of their professional careers added to the establishment of a robust networking. Conclusion: Our group was inspired by those initiatives, and discussed strategies to analyse the professional profiling of women working in different areas of transplantation and organ donation in Brazil, and to identify possible barriers along the development of their careers and transition towards leadership. Therefore, we intend to establish the Brazilian WIT chapter at the next congress of the Brazilian Association of Organ Transplantation, in October 2019 in the city of Campinas, São Paulo, Brazil, as well as to propose a steering committee.

Keywords: Gender Equity; Working Women; Transplantation.

\section{REFERÊNCIAS}

1. https://www.tts-wit.org/

2. Dwyer KM, Clark CJ, MacDonald K, Paraskeva MA, Rogers N, Ryan J, et al. Gender Equity in Transplantation: A Report from the Women in Transplantation Workshop of The Transplantation Society of Australia and New Zealand. Transplantation. 2017;101(10):2266-70

3. Smith KA, Arlotta P, Watt FM, The Initiative on Women in Science and Engineering Working Group, and Susan L. Solomon. Seven Actionable Strategies for Advancing Women in Science, Engineering, and Medicine. Cell Stem Cell. 2015;16(3):221-4.
4. https://www.elsevier.com/data/assets/pdf_file/0008/265661/ ElsevierGenderReport_final_for-web.pdf

5. http://jump.eu.com/studies/Women leaders speak out!

6. http://jump.eu.com/studies/How to get more women into leadership roles? 